\title{
MECHANICAL PROPERTIES, STRUCTURE AND FRACTURE BEHAVIOUR OF ADDITIVE MANUFACTURED FFF-ABS SPECIMENS
}

\author{
0. Gewelber ${ }^{1}$, Y. Rosenthal1, D. Ashkenazi2* ${ }^{*}$ A. Stern ${ }^{1,3}$ \\ ${ }^{1}$ School of Mechanical Engineering, Afeka Academic College of Engineering, Tel Aviv, 6910701, Israel \\ ${ }^{2}$ School of Mechanical Engineering, Tel Aviv University, Ramat Aviv 6997801, Israel \\ ${ }^{3}$ Department of Materials Engineering, Ben-Gurion University of the Negev, Beer Sheva 8410501, Israel \\ * Corresponding author's e-mail address: dana@eng.tau.ac.il
}

\begin{abstract}
The Fused Filament Fabrication (FFF) method is one of the most important additive manufacturing (AM) technologies. This technology is used today with various kinds of thermoplastic materials, including ABS. The present study deals with the flexural strength and axial deflection of ABS specimens versus relative density, to observe the influence of build-orientations, build model and microscopic level defects of these properties. In this study, the mechanical and structural characterization of AM-FFF ABS material was studied by CAD modelling of different orientations, three point bending mechanical testing, visual testing, and multifocal light microscopy observation, including fractography analysis. To that end, three different standard building orientations (Flat, On Edge and Upright) were printed, and each was built in two different angle orientations $\left(-45^{\circ} / 45^{\circ}\right.$ and $\left.0^{\circ} / 90^{\circ}\right)$. Based on the three-point bending testing results, it was found that the specimen with the highest flexural strength was not necessarily the one with the highest deflection. It was also observed that On Edge $0 /+90^{\circ}$ orientations showed a relatively larger flexural strength difference in comparison to other building orientations (Flat and Upright). When the mechanical properties achieved from a bending test next to the building platform were compared to the properties far from the building platform, only a slight difference was found, which means that the flexural strength difference results from the building strategy and it is not related to the specific bending surface. Based on fractography observation, there is a major difference in the mechanical properties and fracture surface appearance, when the samples are bent between the layers (Upright orientation) or when the samples are bent through the layers (Flat and On Edge orientation).
\end{abstract}

KEYWORDS: Additive manufacturing, ABS, CAD model, Fused filament fabrication, Fractography, Mechanical properties, Three-point bend flexural test.

\section{INTRODUCTION}

Additive manufacturing (AM) has been currently gaining ground through the introduction of new manufacturing technologies for complex functional/structural components, needed by specialized industries, including the biomedical and aerospace industries, and many others. The polymer extrusion AM method, commonly referred to as fused deposition modelling (FDM) and formally known as fused filament fabrication (FFF), is based on a thermoplastic fiber fed into a hot-chamber and extruded through a nozzle at its melting temperature [1]-[4]. The extruded semi-liquid polymer filaments cool rapidly to build-chamber temperature, and create a two-dimensional layer on top of the surface of the previously deposited stratum (Fig. 1). Commonly, the FFF parts are printed on a build-platform and the first few layers constitute a support section to assist in the removal of the actual part from the build-plate. At this point, the material is further extruded to print the actual component layer by layer. The total volume of the support material depends upon the contact area between the component and the build-platform, in addition to the overhung portions of the object [3]-[7].

The structural requests for FFF printed components continue to expand, leading to complex loading configurations and requirements for a thorough characterization of printed parts. The FFF technology has inherent weaknesses, such as producing parts with anisotropic mechanical properties; even the same component normally has a 
different mechanical behaviour when printed in different orientations [5]-[9]. To overcome the challenges, many researchers focused on the quality of structural FFF products, which depends on how different settings of process parameters impact the mechanical behaviour of the manufactured end-parts [8], [10]-[12].

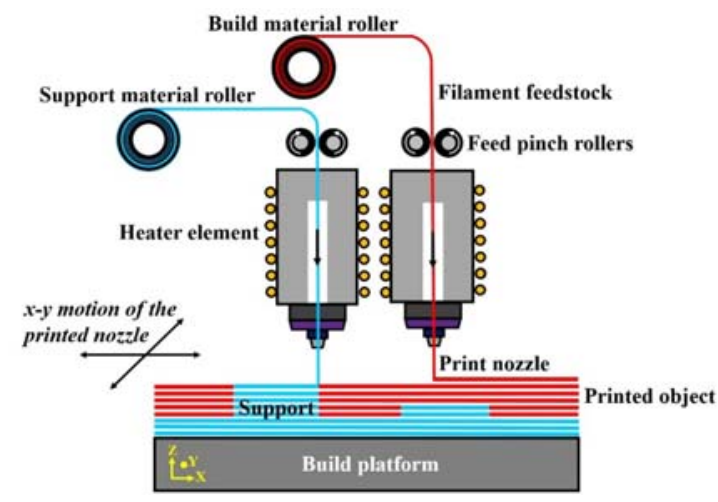

Fig. 1. Schematic representation of the FFF process. The technique is based on a thermoplastic filament fed into the heating chamber and extruded through a print nozzle to create the $3 \mathrm{D}$ components

A partial list of the main FFF process parameters is given below [13]-[18]:

- Build-orientation, build parameters, e.g., layer thickness, infill density, raster pattern, air gaps, number of contours/perimeters.

- Temperature conditions, e.g. environment envelope temperature, extrusion temperature and platform temperature.

Several reports have studied the mechanical properties of FFF produced components and researchers have worked on two directions in attempting to improve the mechanical properties of the end-parts:

- Design for FFF and evaluation of suitability for given applications [9], [15]. Hence, the analysis of the mechanical properties of FFF parts represents an important subject of interest and research.

- Process parameters optimization demonstrated that joint quality between adjacent fibers is mainly determined by the envelope temperature and by variations in temperature conditions within the build-part [3], [14]-[20].

The most commonly studied inexpensive polymer commonly used in combination with the FFF process is ABS $\left[\left(\mathrm{C}_{8} \mathrm{H}_{8}\right)_{\mathrm{x}} \cdot\left(\mathrm{C}_{4} \mathrm{H}_{6}\right)_{\mathrm{y}} \cdot\left(\mathrm{C}_{3} \mathrm{H}_{3} \mathrm{~N}\right)_{\mathrm{z}}\right]$. ABS is an amorphous, thermoplastic glassy polymer which is supplied in the form of a flexible monofiber, fed into a preheated FFF extruder [5]-[12]. The material contains acrylonitrile (A), styrene (S), and polybutadiene (PB); the most common ABS microstructure is made of a continuous and rigid $\mathrm{A}+\mathrm{S}$ phase with compatible $\mathrm{PB}$ inclusions in the range of $0.1 \mu \mathrm{m}$ to $25 \mu \mathrm{m}$ radius [16]. In recent years, the most significant process parameters considered as influencing FFF specimens' tensile, compression, flexural or impact strengths have been studied and discussed [14]-[20].

The studies show that two inherent discontinuities affect the mechanical properties of FFF-ABS components: porosity and defective weldzones. Porosity is inherent to all FFF techniques because the cylindrical warm filament with rounded features is deposited layer by layer, thus creating intra-layer and inter-layer porosity. The intra-layer porosity depends on the raster-pattern; the oval fibers do not stack into perfectly dense bands leading to inter-raster porosity. When building a layer, the bottom of the filament is flattened and the top retains its cylindrical shape; therefore, porosity is created between the rounded filament and the flat bottom of the subsequent layer, forming inter-layer porosity. Miscellaneous voids therefore persist between rasters, both within the layers and between layers [3]-[5], [15]-[20].

The joint between the adjacent filaments takes place due to thermally driven diffusion bonding. Complete bonding takes place when a local volume of material within adjacent fibers, in intimate contact, reaches an appropriate temperature, thus enabling polymer chains to diffuse and entangle across the interface. In fact, the bonding interface is formed between a semi-liquid polymer brought in contact with the previously deposited colder polymer. Lower deposition speeds and higher temperatures can improve the joint-forming diffusion process, but will lead to inferior geometric control, higher roughness, and extended print times. Even when filaments are in intimate contact, the fiber-to-fiber bond strength is unlikely to be comparable to that of the bulk polymer due to the transient nature of the diffusion process; the temperature rapidly decreases in the movement direction of the extrusion head. Defective weld-zones are initiated and found inside the layers (intra-layer defects) and in-between the layers (inter-layer defects). All of the above mentioned FFF parameters affect porosity formation and fiber-to-fiber bonding, and thus influence the mechanical properties of the ABS end-parts.

The increase in commercial manufacturing demands improved the understanding of the mechanical properties of FFF-ABS made parts and the optimization of the FFF printing process for various products. This research, as part of an ongoing project [21]-[23], aims to identify the correlation between flexural strength, deflection and density for different build-orientations and raster patterns. Fractography and failure surface visualization will be presented; the mode of failure will be also discussed.

The present results will be compared to other studies existing in the literature, which are mainly based on special experimental methodology, covering different $3 \mathrm{D}$ printing parameters. 


\section{EXPERIMENTAL PART}

\subsection{ABS Specimens}

ABS plus (STRATASYS) thermoplastic samples were produced on the STRATASYS Dimension Elite 3D (C) printer machine according to the ASTM D790 standard for 3-point bending; their dimensions were $127 \times 12.7 \times 3.2 \mathrm{~mm}$. According to the manufacturer, the ABS plus filament has a glass transition temperature of $108^{\circ} \mathrm{C}$, melt temperature of $\sim 235^{\circ} \mathrm{C}$, a recommended bed temperature of $90-110^{\circ} \mathrm{C}$, the yield tensile strength of $31 \mathrm{MPa}$ and the ultimate tensile strength of $33 \mathrm{MPa}$. The 3D printing parameters defined for this research were solid density (STRATASYS), with a layer thickness of $0.1778 \mathrm{~mm}$. Sample orientation was described by the orthogonal orientation notation as outlined in ISO/ASTM 52921:2013 [24]. In this notation, as shown in figure 2 , sample orientations are identified by a three-letter designation based upon a sample's bounding box being aligned parallel to the $\mathrm{X}, \mathrm{Y}$, and $\mathrm{Z}$ axes. One lists the axis parallel to the longest overall dimension first, followed by the axis parallel to the second longest dimension, and finally followed by the axis parallel to the shortest dimension.
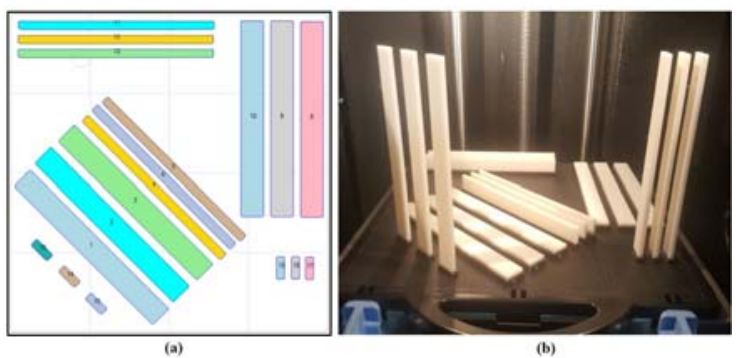

Fig. 3. The experimental test specimens: a) CAD model of the first and second trays (received from the Solidworks program); b) the physical AM-FFF ABS printed specimens of the first and second trays

Twelve three-point bending flexural test ABS specimens were 3D-printed in the third tray (Fig. 4) at two orientations: On Edge $0^{\circ} / 90^{\circ}$ and On Edge $45^{\circ} / 45^{\circ}$ (for each configuration three specimens were 3D-printed and tested). Each of these groups were divided into two: (1) specimens that were bent next to the build platform, and (2) specimens bent far from the build platform, in order to examine whether there was a change in the mechanical properties because of the build platform heating gradient.

The names of the specimens are marked by a numbering designation system. For example, when the specimen is described as 3-On Edge $0^{\circ} / 90^{\circ}-12-\downarrow$ notation, it means that the specimen was printed in tray no. 3, where On Edge is the building orientation, 12 is the number of the specimen in the tray, $\downarrow$ means that the three point bending would be in the area farther from the build platform, and $\uparrow$ means that the
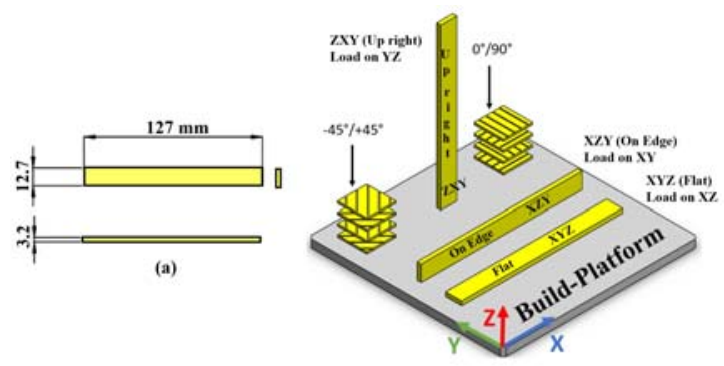

(b)

Fig. 2. The test specimens: a) Schematic illustration of the ABS three-point bending flexural test specimens (front, top and side views); b) the CAD model of the test specimens' location on the tray (isometric view established from the Solidworks program)

XYZ (Flat), XZY (On Edge), and ZXY (Upright) samples were considered for the orientation parameter and $0^{\circ} / 90^{\circ}$, and $-45^{\circ} / 45^{\circ}$ for the raster angle parameter. The CAD model of each test specimen's location on the tray is shown in figure 2.

The first two trays are identical and each of them has eighteen three-point bending flexural test ABS specimens that were 3D-printed at six different orientations (Fig. 3).
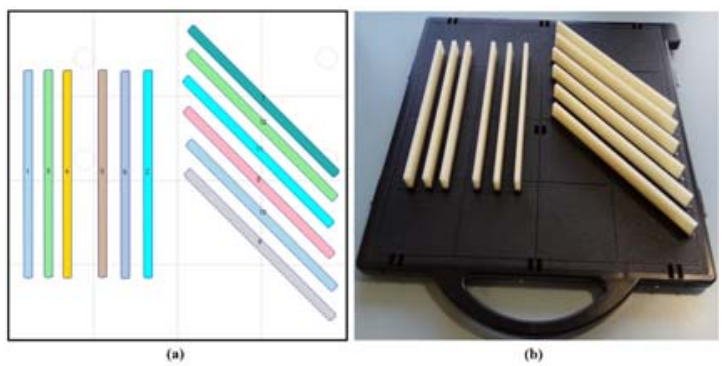

Fig. 4. The experimental test specimens: (a) CAD model of the third tray (received from the Solidworks program); b) the physical AM-FFF ABS printed specimens of the third tray

three point bending specimen would be in the area next to the build platform.

\subsection{Mechanical Properties}

The samples' geometric dimensions are height, width and length. Height and width were measured by a micrometer with an error of $0.01(\mathrm{~mm})$; the length was measured by a digital caliper with an error of $0.02(\mathrm{~mm})$. The mass was weighed by an MRC ABS220-C2 analytical balance with an error of 0.0001(gr). Volume was obtained by multiplying the height, width and length; the volume was then divided by the mass in order to get the absolute density. Relative density was calculated by dividing the absolute density by the supplier's provided density, which is $1.04(\mathrm{gr} / \mathrm{cc})$. The flexural strength of a material is defined as its ability to resist deformation under load 
and it is calculated by using the maximum applied load. For materials that deform significantly but do not break, the load at yield, typically measured at 5\% deformation/strain of the outer surface, is reported as flexural yield strength.

A universal MTS testing machine (Model No. E43) was used, equipped with a three-point bending fixture and a deflection gage (Fig. 5a) to test the mechanical properties. The bending test presents two parameters (Fig. 5b): (1) axial deflection with an error of $0.02(\mathrm{~mm})$; and (2) force in units of Newton $0.5 \%$ error. The applied (initial load was $15[\mathrm{~N}]$ and the maximal load was up to $100-309[\mathrm{~N}])$. The crosshead velocity used for all test specimens was $0.5 \mathrm{~mm} / \mathrm{min}$. Flexural strength was calculated by the formula:

$$
\sigma_{b}=\frac{3 \cdot F \cdot L}{2 \cdot b \cdot h^{2}}
$$

where $\mathrm{F}$ is the applied force, $\mathrm{L}$ is the distance between the two anvils, and $\mathrm{b}$ and $\mathrm{h}$ are the measured dimensions of the specimen's cross-section.
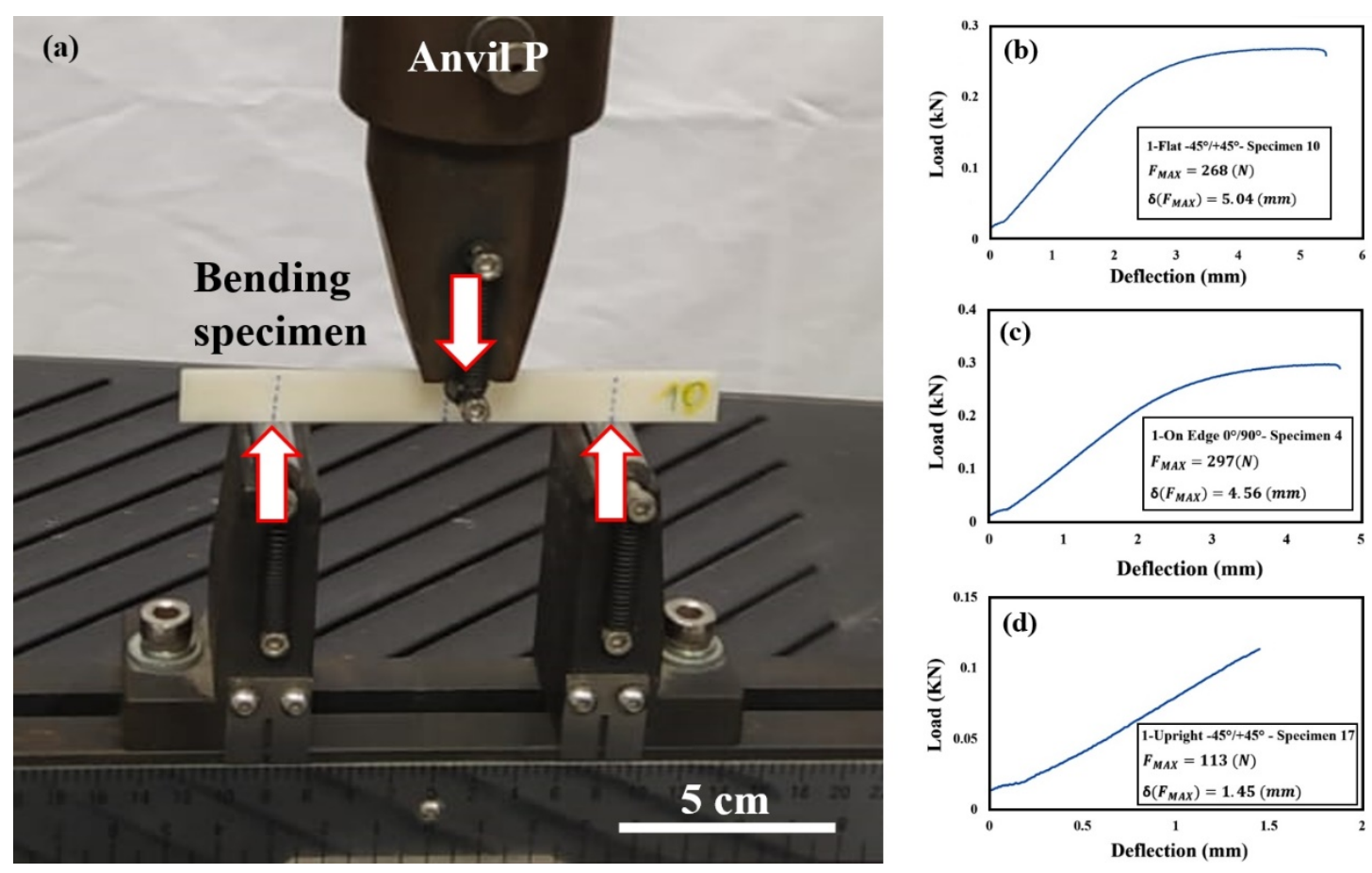

Fig. 5. The experimental system: a) the three-point bending fixture including the three-point bending specimen; b)-d) the experimental results of the deflection $(\mathrm{mm})$ vs. the load $(\mathrm{kN})$ for three different specimens

Visual testing (VT) inspection combined with multifocal 3D digital light microscopy (LM, HIROX RH-2000 instrument) observation were performed following the mechanical testing, to observe the quality of the printed surfaces, including possible defects, as well as to examine the fracture surface morphology ("ductile", "brittle" or "mixed mode").

\section{RESULTS AND DISCUSSION}

\subsection{Mechanical Properties}

The three-point bending experimental results of the relative density $\rho_{\mathrm{r}}(\%)$ vs. axial deflection $\delta(\mathrm{mm})$ and relative density $\rho_{\mathrm{r}}(\%)$ vs. flexural strength $\sigma(\mathrm{MPa})$ for Flat $0^{\circ} / 90^{\circ}$, Flat $-45^{\circ} / 45^{\circ}$, On Edge $0^{\circ} / 90^{\circ}$, On Edge $-45^{\circ} / 45^{\circ}$, Upright $0^{\circ} / 90^{\circ}$ and Upright $45^{\circ} / 45^{\circ}$ specimens are shown in figure 6 , and all orientations are included in table 1.
When the average relative densities of the different building orientations were compared, it was revealed that the average Upright samples were within the range of 88.67-92.09 $\rho_{\mathrm{r}}(\%)$, whereas the average Flat and On Edge samples were within the range of 94.41-98.10 $\rho_{\mathrm{r}}(\%)$, and the On Edge $-45^{\circ} / 45^{\circ}$ orientation was the densest one.

The highest flexural strength orientation was measured in the On Edge $0^{\circ} / 90^{\circ}$, and the weakest ones were measured in the Upright $0^{\circ} / 90^{\circ}$ and Upright $-45^{\circ} / 45^{\circ}$ (at least two times smaller). The remaining results are slightly below the On Edge $0^{\circ} / 90^{\circ}$ orientation. These differences between the flexural strength results may be explained by the fact that the Upright samples were broken immediately, without any warning, while the Flat and On Edge fractures occurred after plastic deformation, which indicates a ductile fracture based on metallurgical terms. 
When the deflections of different groups of the same orientation are compared, it can be seen that no orientation is dominant. This can be explained by the uneven melting distribution of the ABS fibers, resulting in uneven deflections and structural defects that cause longer movement during the bending test.

The numerical data from the first two trays correlates well with the results of a preliminary experiment conducted two years ago (groups start with the letter ' $A$ ', tables 1 and 2), using the same printer machine and the same printing parameters; however, a different filament of the same kind of material was applied during the preliminary experiment. The On Edge average properties of the ABS 3D-printed three-point bending experimental results are shown in table 1, and the average properties for all orientations of the three-point bending experimental results are shown in table 2 .

The third tray was designed and printed in order to check whether there was a difference in the mechanical properties, when the bend was next to the build platform or the farther from the build platform. To that end, twelve samples of On Edge of two orientations were printed: (1) On Edge $0^{\circ} / 90^{\circ}$, and (2) On Edge $-45^{\circ} / 45^{\circ}$. Each of those groups was divided into two: (1) bent next to the build platform (designated by $\uparrow$ ), and (2) bent far from the build platform (designated by $\downarrow$ ).

Table 1. All orientation average properties of the ABS 3D-printed three-point bending experimental results

\begin{tabular}{|l|c|c|c|}
\hline \multicolumn{1}{|c|}{ Specimens } & $\begin{array}{c}\mathrm{r}_{\mathrm{r}} \\
{[\%]}\end{array}$ & $\begin{array}{c}\boldsymbol{\delta} \\
{[\mathbf{m m}]}\end{array}$ & $\begin{array}{c}\boldsymbol{\sigma} \\
{[\mathbf{M P a}]}\end{array}$ \\
\hline 1-On Edge $-45^{\circ} /+45^{\circ}$ avg & 98.10 & 3.85 & 65.34 \\
2-On Edge $-45^{\circ} / 45^{\circ}$ avg & 97.83 & 3.67 & 63.30 \\
A-On Edge $-45^{\circ} / 45^{\circ}$ avg & 94.49 & 4.29 & 63.67 \\
3-On Edge $-45^{\circ} /+45^{\circ}$ & 94.53 & 5.96 & 66.05 \\
3-On Edge $0^{\circ} / 90^{\circ}$ & 95.60 & 5.05 & 70.11 \\
1-On Edge $0^{\circ} / 90^{\circ}$ avg & 97.94 & 4.63 & 71.27 \\
2-On Edge $0^{\circ} / 90^{\circ}$ avg & 97.14 & 4.75 & 70.03 \\
A-On Edge $0^{\circ} / 90^{\circ}$ avg & 94.39 & 4.65 & 68.15 \\
1-Flat $-45^{\circ} / 45^{\circ}$ avg & 94.41 & 5.17 & 61.83 \\
2-Flat $-45^{\circ} / 45^{\circ}$ avg & 95.92 & 5.62 & 64.24 \\
1-Flat $0 \% / 90^{\circ}$ avg & 95.72 & 3.33 & 58.92 \\
2-Flat $0^{\circ} / 90^{\circ}$ avg & 95.35 & 3.20 & 58.42 \\
1-Upright $-45^{\circ} /+45^{\circ}$ avg & 92.09 & 1.42 & 26.56 \\
2-Upright $-45^{\circ} /+45^{\circ}$ avg & 90.17 & 1.43 & 25.32 \\
A-Right $-45^{\circ} / 45^{\circ}$ avg & 89.12 & 1.12 & 22.24 \\
1-Upright $0^{\circ} / 90^{\circ}$ avg & 90.95 & 1.45 & 24.39 \\
2-Upright $0^{\circ} / 90^{\circ}$ avg & 91.39 & 1.54 & 23.94 \\
A-Upright $0^{\circ} / 90^{\circ}$ avg & 88.67 & 1.18 & 24.70 \\
\hline
\end{tabular}

Group 3-On Edge $-45^{\circ} / 45^{\circ}-\uparrow$ with a range of 94.36-95.90 percentage of relative density, 63.85$69.40(\mathrm{MPa})$ flexural strength, and 4.50-7.20 (mm) axial deflection. Group 3-On Edge $-45^{\circ} /+45^{\circ}-\downarrow$ with a range of 93.11-94.30 percentage of relative density, 64.85-66.52 (MPa) flexural strength and 5.65-6.13 $(\mathrm{mm})$ axial deflection.

Table 2. On Edge orientation average properties of the ABS 3D-printed three-point bending experimental results

\begin{tabular}{|l|c|c|c|}
\hline \multicolumn{1}{|c|}{ Specimens } & $\begin{array}{c}\mathrm{r}_{\mathrm{r}} \\
{[\%]}\end{array}$ & $\begin{array}{c}\boldsymbol{\delta} \\
{[\mathbf{m m}]}\end{array}$ & $\begin{array}{c}\boldsymbol{\sigma} \\
{[\mathbf{M P a}]}\end{array}$ \\
\hline 1-On Edge $-45^{\circ} / 45^{\circ}$ avg & 98.10 & 3.85 & 65.34 \\
2-On Edge $-45^{\circ} / 45^{\circ}$ avg & 97.83 & 3.67 & 63.30 \\
A-On Edge $-45^{\circ} / 45^{\circ}$ avg & 94.49 & 4.29 & 63.67 \\
3-On Edge $-45^{\circ} / 45^{\circ}-\uparrow$ avg & 95.18 & 5.82 & 66.25 \\
3-On Edge $-45^{\circ} / 45^{\circ}-\downarrow$ avg & 93.88 & 6.10 & 65.85 \\
1-On Edge $0^{\circ} / 90^{\circ}$ avg & 97.94 & 4.63 & 71.27 \\
2-On Edge $0^{\circ} / 90^{\circ}$ avg & 97.14 & 4.75 & 70.03 \\
A-On Edge $0 \% / 90^{\circ}$ avg & 94.39 & 4.65 & 68.15 \\
3-On Edge $0^{\circ} / 90^{\circ}-\uparrow$ avg & 96.04 & 4.99 & 70.41 \\
3-On Edge $0^{\circ} / 90^{\circ}-\downarrow$ avg & 95.15 & 5.11 & 69.82 \\
\hline
\end{tabular}

The three-point bending experimental results of relative density $\rho_{\mathrm{r}}(\%)$ vs. axial deflection $\delta(\mathrm{mm})$ and relative density $\rho_{\mathrm{r}}(\%)$ vs. flexural strength $\sigma(\mathrm{MPa})$ of the ABS 3D-printed three-point bending On Edge orientation are shown in figure 7.

Group 3-On Edge $0^{\circ} / 90^{\circ}-\uparrow$ with a range of 95.24-96.55 percentage of relative density, 69.62$71.69(\mathrm{MPa})$ flexural strength, and 4.43-5.28 (mm) axial deflection. Group 3-On Edge $0^{\circ} / 90^{\circ}-\downarrow$ with a range of 94.34-95.43 percentage of relative density, $68.82-70.70(\mathrm{MPa})$ flexural strength and $4.89-5.53$ $(\mathrm{mm})$ axial deflection.

The overlapping values and the On Edge average results seen in table 1 lead to the conclusion that the difference in the mechanical properties results from the building orientation and not from the different area of bending.

\subsection{Fractography and Fracture Visualization}

A simple CAD model was prepared to visualize and predict the expected fracture surface morphology of the specimens prior to the bending test. The idealised model was based on average filaments and layer dimensions, which in turn were based on the received fractography images, with an elliptic filament crosssection $(0.18 \mathrm{~mm}$ by $0.31 \mathrm{~mm})$. These filament dimensions were used to draw the horizontal and vertical layers. This procedure was applied to create visualization models for two printed geometries, Upright $-45^{\circ} / 45^{\circ}$ and Upright $0^{\circ} / 90^{\circ}$, which were compared to the actual LM observation of the fracture faces (Fig. 8a, 8b). Some disadvantages of the visualization model were observed, i.e., (a) the model described a perfect surface fracture with no filament melting and/or asymmetric filament cross section; all were clearly visible in the fractography examinations. 


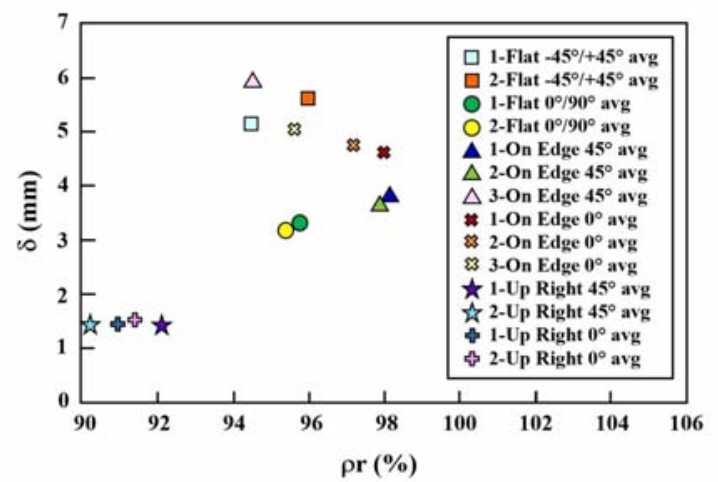

(a)

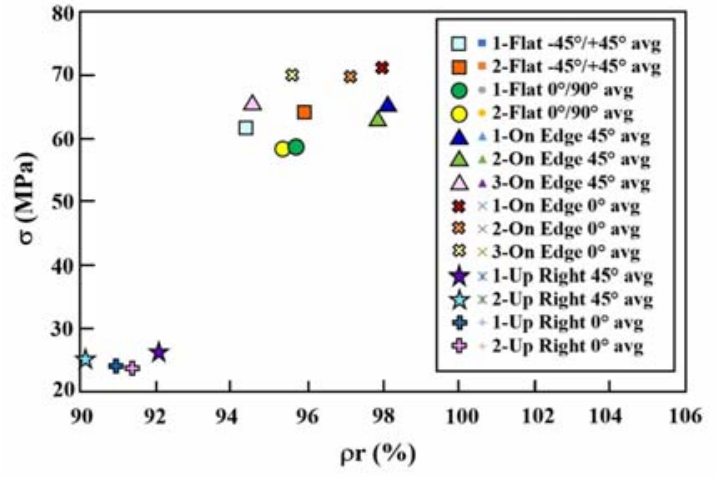

(b)

Fig. 6. ABS 3D-printed three-point bending experimental results of the Flat, On Edge and Up-right configurations: a) density vs. deflection; b) density vs. flexural strength

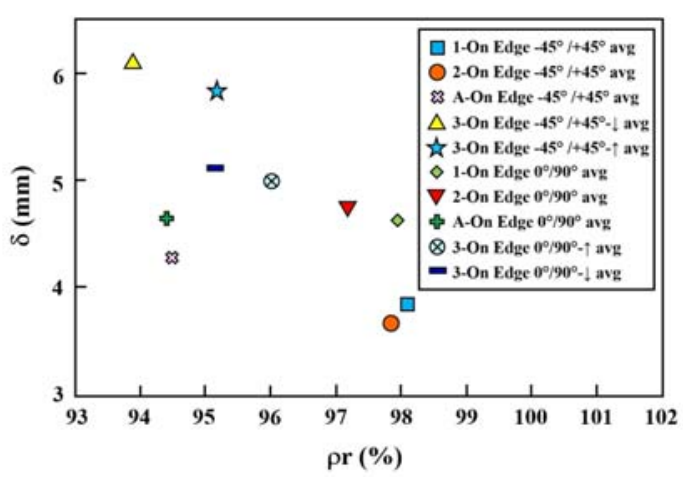

(a)

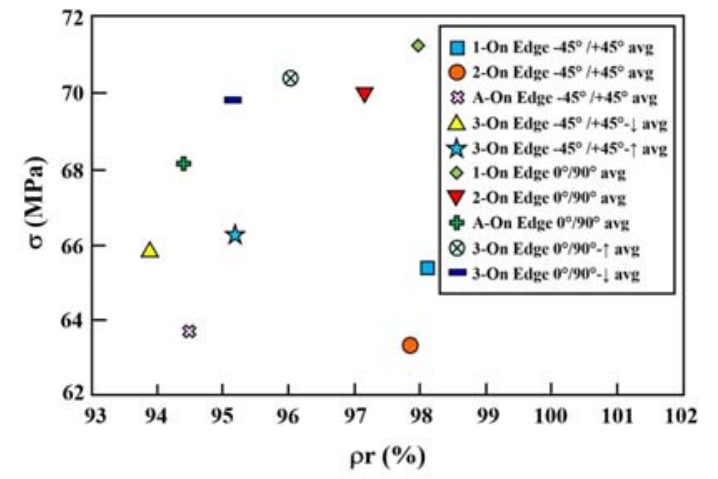

(b)

Fig. 7. ABS 3D-printed three-point bending experimental results of the On Edge orientation: a) density vs. deflection; b) density vs. flexural strength. Arrow up refers to bending in area with contact to tray, arrow down refers to bending at the area where the building of the specimen was finished

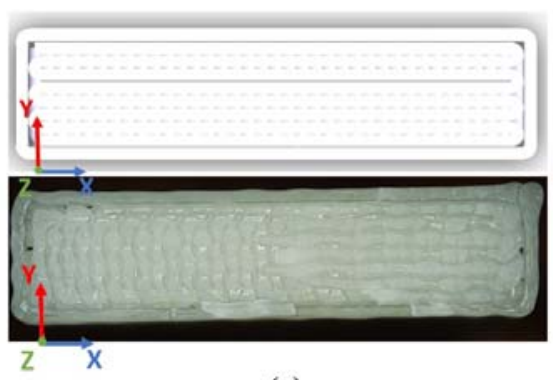

(a)

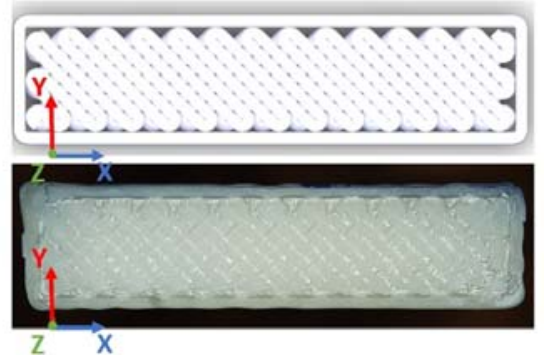

(b)

Fig. 8. a) Upright $0^{\circ} / 90^{\circ} \mathrm{CAD}$ model (upper image) and LM photo (lower image); b) Upright $-45^{\circ} /+45^{\circ} \mathrm{CAD}$ model (upper image) and LM photo (lower image)

The visualization model used emphasizes the need for a proper machine G-code for pre-print design and the need for well-conceived dimensional planning for the prevention of defects and cavities in the printed structure.

During the three-point bending test, there is a major difference in the mechanical properties and fracture surface topography when the samples are bent between the layers (Upright orientation) and when the samples are bent through the layers (Flat and On Edge orientation). Figure 9e shows an On Edge $-45^{\circ} / 45^{\circ}$ orientation of a single cut (crack propagation during the bending test) through the layer, whereas figure 9f shows an On Edge $0^{\circ} / 90^{\circ}$ orientation of one half vertical weaved layer and the second half horizontal weaved layer. In contrast to the 
Upright samples, the Flat and One Edge orientations (Fig. 9a-d) result in rough surface topography, with height differences between the broken material layers, which indicates impure fracture topography with correlation to the mechanical data.

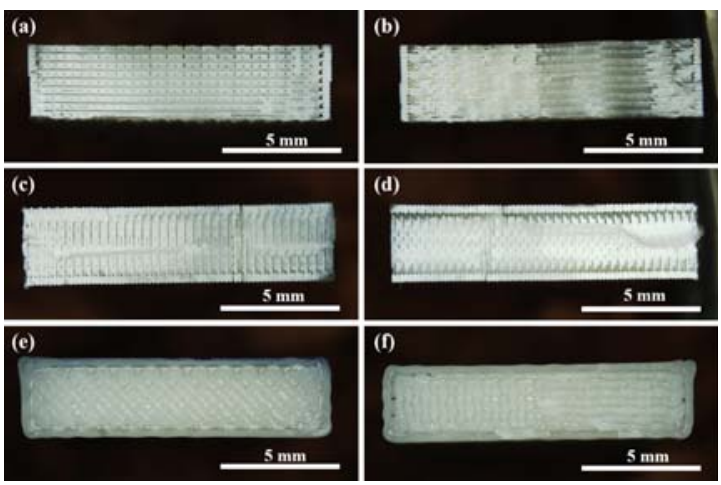

Fig. 9. The fracture surfaces of the ABS printed specimens after the three-points bending tests:

a) 1-Flat $0^{\circ} / 90^{\circ}-2$ specimen; b) 1-Flat $-45^{\circ} /+45^{\circ}-10$ specimen; c) 1-On Edge $-45^{\circ} / 45^{\circ}-12$ specimen;

d) 1-On Edge $0^{\circ} / 90^{\circ}-4$ specimen; e) 1-Upright $-45^{\circ} \%$ $45^{\circ}-17$ specimen; f) 1 Upright $0^{\circ} / 90^{\circ}-14$ specimen

The present flexural results cover a wide range of FFF build-up strategies and printing positions [25]; for alike specimens the data presented here is fairly similar to that published in other research papers, as each describes only some of the relevant parameters $[11],[26]-[27]$.

\section{SUMMARY}

In this study, the mechanical and structural characterizations of AM-FFF ABS material were performed to broaden the understanding of flexural properties. Thus, specimens with rectangular crosssections were 3D-printed in various densities with three different building orientations (Flat, On Edge and Upright), and two different angle orientations ($45^{\circ} / 45^{\circ}$ and $0^{\circ} / 90^{\circ}$ ), and were characterized by the three-point-bend test, VT, and multifocal LM, including fractography analysis, to study the influence of build-orientations, build model and microscopic level defects on the mechanical properties. The results revealed that the specimen with the highest amount of flexural strength was not necessarily the one with the highest deflection. It was also found that the specimens that were printed parallel to the $x-y$ plane of the tray (On Edge $0^{\circ} / 90^{\circ}$ ) exhibited relatively larger flexural strength differences than other building orientations (On Edge and Up-right). It was also observed that there was no major difference in the data value when the bending test was performed next to the building surface or far from it. Based on the fractography results, there is a major difference in the mechanical properties and fracture surface appearance, when the bending samples are bent between the layers (Upright orientation) and when they are bent through the layers (Flat and On Edge orientation).

\section{ACKNOWLEDGEMENTS}

The authors would like to acknowledge the technical assistance of N. Dresler and A. Solomon from the Afeka College of Engineering. Thanks are due to S. Maman, A. Ulanov and N. Bilenkin, Afeka Academic College of Engineering, for their engineering assistance. Thanks are also due to M. Cohen and D. Cvikel (Haifa University), and the assistance provided by I. Zilberman (Afeka Academic College of Engineering) is greatly appreciated. The authors are also grateful to Barbara Doron for the English editing.

\section{REFERENCES}

[1] Klippstein H., Diaz De Cerio Sanchez A., Hassanin H., Zweiri Y., Seneviratne, L., Fused deposition modeling for unmanned aerial vehicles (UAVs): A Review, Advanced Engineering Materials vol. 20, iss. 2, 2018, p. 1700552.

[2] Herzog D., Seyda V., Wycisk E., Emmelmann C., Additive manufacturing of metals. Acta Materialia, vol. 117, 2016, pp. 371392.

[3] Popescu D., Zapciu A., Amza C., Baciu F., Marinescu R., FDM process parameters influence over the mechanical properties of polymer specimens: A review, Polymer Testing, vol. 69, 2018, pp. 157-166.

[4] Dizon J. R. C., Espera Jr. A. H., Chen Q., Advincula R. C., Mechanical characterization of 3D-printed polymers, Additive Manufacturing, vol. 20, 2018, pp. 44-67.

[5] Garg A., Bhattacharya A., Batish A., Chemical vapor treatment of ABS parts built by FDM: Analysis of surface finish and mechanical strength, The International Journal of Advanced Manufacturing Technology, vol. 89, 2017, pp. 2175-2191.

[6] Hart K. R., Wetzel E. D., Fracture behavior of additively manufactured acrylonitrile butadiene styrene (ABS) materials, Engineering Fracture Mechanics, vol. 177, 2017, pp. 113.

[7] McLouth T. D., Severino J. V., Adams P. M., Patel D. N., Zaldivar R. J., The impact of print orientation and raster pattern on fracture toughness in additively manufactured ABS, Additive Manufacturing 18, 2017, pp. 103-109.

[8] Rodriguez J. F., Thomas J. P., Renaud J. E., Design of fuseddeposition ABS components for stiffness and strength, Journal of Mechanical Design 125.3, 2003, 125, pp. 545-551.

[9] Hernandez R., Slaughter D., Whaley D., Tate J., Asiabanpour B., Analyzing the tensile, compressive, and flexural properties of $3 D$ printed ABS $P 430$ plastic based on printing orientation using fused deposition modeling. In: 27th Annual International Solid Freeform Fabrication Symposium, Austin, TX, 2016, pp. 939-950.

[10] Rankouhi B., Javadpour S., Delfanian F., Letcher T., Failure analysis and mechanical characterization of $3 D$ printed ABS with respect to layer thickness and orientation, Journal of Failure Analysis and Prevention 16, 2016, 467-481.

[11] Tymrak B. M., Kreiger M., Pearce J. M., Mechanical properties of components fabricated with open-source 3-D printers under realistic environmental conditions, Materials \& Design 58, 2014, pp. 242-246.

[12] Wu W., Geng P., Li G., Zhao D., Zhang H., Zhao J., Influence of layer thickness and raster angle on the mechanical properties of $3 D$-Printed PEEK and a comparative mechanical study between PEEK and ABS, Materials, vol. 8, 2015, pp. 58345846.

[13] Ulu E., Korkmaz E., Yay K., Burak Ozdoganlar O., Burak Kara L., Enhancing the structural performance of additively manufactured objects through build orientation optimization, Journal of Mechanical Design 137.11, 2015, p. 
111410.

[14] Durgun I., Ertan R., Experimental investigation of FDM process for improvement of mechanical properties and production cost, Rapid Prototyping Journal 20, 2014, pp. 228-235.

[15] Somireddy M., Czekanski A., Singh C. V., Development of constitutive material model of $3 D$ printed structure via FDM, Materials Today Communications 15, 2018, pp. 143-152.

[16] Hibbert K., Warner G., Brown C., Ajide O., Owolabi G., Azimi A., The Effects of Build Parameters and Strain Rate on the Mechanical Properties of FDM 3D-Printed Acrylonitrile Butadiene Styrene, Open Journal of Organic Polymer Materials, 9.1, 2019, pp. $1-27$.

[17] Dana H. R., Barbe F., Delbreilh L., Azzouna M. B., Guillet A., Breteau T., Polymer additive manufacturing of ABS structure: Influence of printing direction on mechanical properties, Journal of Manufacturing Processes 44, 2019, pp. 288-298.

[18] Górski F., Wichniarek R., Kuczko W., Zawadzki P., Buń P., Strength of ABS parts produced by Fused Deposition Modelling technology-a critical orientation problem, Advances in Science and Technology Research Journal vol. 9, iss. 26, 2015, pp. 12-19.

[19] Conway K. M., Pataky G. J., Crazing in additively manufactured acrylonitrile butadiene styrene, Engineering Fracture Mechanics 211, 2019, pp. 114-124.

[20] Jo W., Kwon O. C., Moon M. W., Investigation of influence of heat treatment on mechanical strength of FDM printed 3D objects, Rapid Prototyping Journal, vol. 24, iss. 3, 2018, pp. 637644.

[21] Shabat D., Rosenthal Y., Ashkenazi D., Stern A., Mechanical and Structural Characteristics of Fused Deposition
Modelling ABS Material, Annals of "Dunarea de Jos" University, Fascicle XII, Welding Equipment and Technology, vol. 28, 2017, pp. 16-24.

[22] Solomon A., Rosenthal Y., Ashkenazi D., Stern A., Structure and mechanical behavior of additive manufactured fused deposition modeling ABS, Annals of "Dunarea de Jos" University of Galati, Fascicle XII, Welding Equipment and Technology, vol. 29, 2018, pp. 47-56.

[23] Rosenthal Y., Apelstein Y., Rosenthal I., Ashkenazi D., Stern A., On laser powder-bed fusion of additively manufactured AlSi10Mg alloy: tensile properties and structure characterization, Annals of "Dunarea de Jos" University of Galati, Fascicle XII, Welding Equipment and Technology 30, 2019, pp. 5-12.

[24] *** ISO/ASTM 52921-2013(E), Standard terminology for additive manufacturing - Coordinate systems and test methodologies, 2013, p. 14.

[25] Dawoud M., Taha I., Ebeid S.J., Mechanical behaviour of ABS: An experimental study using FDM and injection moulding techniques, Journal of Manufacturing Processes, vol. 21, 2016, pp. $39-45$

[26] Reis P.N.B., Gorbatikh L., Ivens J., Lomov S.V., Strain-rate sensitivity and stress relaxation of hybrid self-reinforced polypropylene composites under bending loads, Composite Structures vol. 209, 2019, pp. 802-810.

[27] Torrado A.R., Shemelya C.M., English, J.D., Lin, Y., Wicker, R.B., Roberson, D.A., Characterizing the effect of additives to ABS on the mechanical property anisotropy of specimens fabricated by material extrusion $3 D$ printing, Additive Manufacturing, vol. 6, 2015, pp. 16-29. 